Monatsschr Kinderheilkd 2022 · 170:10-12 https://doi.org/10.1007/s00112-021-01371-x Angenommen: 4. November 2021

(C) The Author(s), under exclusive licence to Springer Medizin Verlag GmbH, ein Teil von Springer Nature 2021

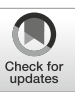

\section{Seltene Erkrankungen}

\author{
Georg F. Hoffmann' · Helge Hebestreit ${ }^{2}$ \\ ${ }^{1}$ Zentrum für Kinder- und Jugendmedizin und Zentrum für Seltene Erkrankungen, Universitätsklinikum \\ Heidelberg, Heidelberg, Deutschland \\ ${ }^{2}$ Zentrum für Seltene Erkrankungen - Referenzzentrum Nordbayern und Universitäts-Kinderklinik, \\ Würzburg, Deutschland
}

Die weitaus meisten der mehr als 8000 bekannten seltenen Erkrankungen manifestieren sich im Kindes- und Jugendalter; ein Großteil ist genetisch bedingt. Unter der Annahme, dass ca. 4-6\% der deutschen Bevölkerung an einer seltenen Erkrankung leiden - im Kindes- und Jugendalter dürfte der Anteil wesentlich höher sein -, besteht bei ca. 700.000 der 13,75 Mio. unter 18Jährigen in Deutschland eine solche Erkrankung. Viele dieser Erkrankungen betreffen mehrere Organsysteme und verlaufen chronisch-progredient.

Seltene Erkrankungen haben damit sowohl in der ambulanten wie auch der stationären pädiatrischen Versorgung eine große Bedeutung. In der Betreuung stellen sich dem Kinder- und Jugendarzt jedoch verschiedene Herausforderungen in Bezug auf Diagnosefindung, spezialisierte und multiprofessionelle Betreuung sowie Überleitung in die Erwachsenenmedizin.

\section{I) Seltene Erkrankungen sind in der} Pädiatrie bundesweit bei ca. 700.000 Betroffenen von großer Bedeutung

Das Nationale Aktionsbündnis für Menschen mit Seltenen Erkrankungen hat 2013 unter intensiver Beteiligung der Betroffenenverbände einen Nationalen Aktionsplan vorgestellt, in dem in verschiedenen Maßnahmenvorschlägen Lösungsansätze für die oben genannten Herausforderungen entwickelt und konsentiert wurden. Das Innovationsfondsprojekt "TRANSLATENAMSE" hat - finanziert durch den Gemeinsamen Bundesausschuss (G-BA) in den zurückliegenden Jahren sämtliche Maßnahmen ausgearbeitet, über 3 Jahre angewandt und evaluiert. Die Möglichkeiten einer verbesserten Diagnosestellung für Kinder und Jugendliche mit seltenen Erkrankungen mit einer eindeutigen Klärung von über $30 \%$ zuvor unklaren Krankheitsbildern sind in diesem Heft von Krude et al. dargestellt.

In Bezug auf die Diagnosestellung seltener Erkrankungen gewinnt die molekulargenetische Diagnostik mithilfe des Next Generation Sequencing gerade in der Kinder- und Jugendmedizin eine zunehmende Bedeutung. Scholz et al. fassen in ihrem Beitrag den aktuellen Stand, aber auch die Herausforderungen der Exomsequenzierung zusammen.

Die Anforderungen an eine strukturierte Transition von Kindern und Jugendlichen in die Erwachsenenversorgung werden in einem Beitrag von Grasemann et al. beleuchtet, der auch den damit verbundenen Ressourcenverbrauch adressiert.

Die wichtige Rolle der Betroffenen und der Selbsthilfe bei der Entwicklung von Versorgungsstrukturen für Menschen mit seltenen Erkrankungen stellen Stephan Kruip (Mitglied des Deutschen Ethikrats, Vorsitzender des Mukoviszidose e.V. und selbst Betroffener), Mirjam Mann und Christine Mundlos (beide Allianz Chronischer Seltener Erkrankungen) dar.

Die besondere auch psychische Belastung der Kinder- und Jugendlichen mit einer seltenen Erkrankung und ihrer Angehörigen sowie neue Formen der Versorgung werden in dem Beitrag von Wiegand-Grefe et al. zum Innvationsfondsprojekt Care-Fam-Net thematisiert.

Dieser Überblick wird durch die Originalarbeiten von Choukair et al. sowie Grasemann et al. ergänzt, die die Ergebnisse des Projekts TRANSLATE-NAMSE zur Entwicklung eines generischen klinischen Versorgungspfads von der Bestätigung ei- 
Hier steht eine Anzeige.

黑 Springer 
ner Verdachtsdiagnose bis zur Etablierung der multiprofessionellen Versorgung für Kinder- und Jugendliche mit seltenen Erkrankungen bzw. die Anforderungen an eine strukturierte Transition von Kindern und Jugendlichen in die Erwachsenenversorgung beleuchten.

\section{Korrespondenzadresse}

\section{Prof. Dr. Georg F. Hoffmann}

Zentrum für Kinder- und Jugendmedizin und Zentrum für Seltene Erkrankungen, Universitätsklinikum Heidelberg Im Neuenheimer Feld 430, 69120 Heidelberg Deutschland georg.hoffmann@med.uni-heidelberg.de

\section{Prof. Dr. Helge Hebestreit}

Zentrum für Seltene Erkrankungen -

Referenzzentrum Nordbayern und UniversitätsKinderklinik

Josef-Schneider-Str. 2, 97080 Würzburg,

Deutschland

hebestreit_h@ukw.de

Interessenkonflikt. G.F. Hoffmann und H. Hebestreit geben an, dass kein Interessenkonflikt besteht.

\section{A. Seidel, Schneider, P. Steinborn \\ Praxishandbuch Autismus}

ICF-orientiertes Arbeiten: Beratung, Diagnostik und Unterstützungsplanung für Menschen mit Autismus-Spektrum-Störung

\section{Weinheim: Beltz Juventa 2021, 1. Aufl., 204 S., (ISBN: 978-3-7799-6602-9), Broschiert, 24,95 EUR}

In den letzten Jahren ist in der Versorgung das Bewusstsein gewachsen, dass der diagnostische Prozess bei Störungen der neuronalen Entwicklung, z.B. beim Autismus, eine eingehende Analyse der Funktionstüchtigkeit im Alltag beinhalten muss. Für das ganzheitliche Verständnis einer Person, ihrer aktuellen Situation und Leistungsfähigkeit in einem gegebenen Kontext sind die gängigen Nomenklaturen des DSM-5 und der ICD-10/11 allein unzureichend.

Die Internationale Klassifikation der Funktionsfähigkeit, Behinderung und Gesundheit (ICF) der WHO erlaubt dagegen eine standardisierte und holistische Erfassung einer Person unter Einbeziehung von Köperstrukturen- und -funktionen, Aktivitäten und Teilhabe, anderen Personeneigenschaften sowie hinderlichen und erleichternden Umweltfaktoren. Mit der Diagnostik nach ICF kann die Grundlage für zielgerichtete Intervention gelegt werden, wobei auch Stärken des Individuums und Verbesserungspotentiale in der Umwelt der Person berücksichtigt werden können. Mittlerweile wird die Anwendung der ICF nicht nur empfohlen, sondern ihr Gebrauch ist nach den gesetzlichen Änderungen im Rahmen des Bundesteilhabegesetzes für die Bedarfsermittlung nach § 118 SGB IX auch notwendig, um Fördermaßnahmen der Eingliederungshilfe zu erhalten.

Das Buch Praxishandbuch Autismus ist das erste Lern- und Lehrbuch, das gezielt versucht, Fachkräften nicht nur die Philosophie der ICF näher zu bringen, sondern auch seine praktische Anwendung. Dies ist sehr wertvoll, da die Implementierung der ICF im professionellen Alltag in der Regel noch immer eine große Herausforderung darstellt. Während das bio-psycho-soziale Modell der ICF von vielen begrüßt wird, ist die umfassende Klassifikation mit ihren mehr als 1675 Kategorien ohne weitere Spezifikation und Hilfen nur schwerlich effektiv einzusetzen.
Um Praktiker beim Einsatz der ICF zu unterstützen, bietet das Buch konkrete Beispiele für die ICF-basierte Gestaltung von Erhebungsverfahren und Formulierung von Fördermaßnahmen. Die Beispiele werden jeweils anhand von Fällen illustriert und mit den gesetzlichen Rahmenbedingungen verknüpft.

Besonders herauszuheben ist, dass das Buch auch über die sogenannten ICF Core Sets für Autismus informiert. Die Core Sets sind eine von der WHO autorisierte Auswahl von ICF-Kategorien, die in hohem Maß sensitiv und spezifisch für Autismus sind. Die Core Sets wurden im Rahmen einer Serie von wissenschaftlichen Arbeiten entwickelt und stellen eine direkte Verbindung zwischen der Diagnose Autismus und der Arbeit mit ICF bei Personen mit dieser Diagnose her.

Mit diesem Buch wird ein großer Schritt in Richtung Anwenderfreundlichkeit und Akzeptanz der ICF bei Autismus gemacht. Für alle, die sich beruflich mit Diagnostik und Intervention bei Autismus beschäftigen, ist seine Lektüre daher unbedingt zu empfehlen.

Prof. Dr. Sven Bölte, Karolinska Institutet, Stockholm, Schweden 